\title{
PESO DE NASCIMENTO COMO PREDITOR PARA A GRAVIDADE DA LESÃO DA SUBSTÂNCIA BRANCA CEREBRAL NEONATAL
}

\author{
Nayara Argollo', Ines Lessa², Suely Ribeiro ${ }^{3}$, Katiusha C. Abreu, , Juliana M.S.Pinto4, \\ Raquel P. Faria ${ }^{4}$, Tatiana G. Telles 4 , Gabriel B. Santos ${ }^{4}$
}

RESUMO - Para analisar a associação entre fatores natais com a gravidade da lesão da substância branca (LSB) cerebral neonatal, controlando o peso de nascimento, identificaram os neonatos pela ultra-sonografia craniana, que foram divididos em: aqueles com evolução da LSC para resolução da imagem ao ultra-som (menor gravidade) e, aqueles que evoluiram com formação de cistos e/ou ventriculomegalia e/ou hemorragia (maior gravidade). Doze variáveis (hiponatremia, anemia, infecção, retinopatia, displasia broncopulmonar, hipoalbuminemia, persistência do canal arterial, audiometria alterada, desconforto respiratório precoce, peso de nascimento $<2500 \mathrm{~g}$, peso por categoria e prematuridade) tiveram diferenças entre os dois grupos $(p<0,05)$, sendo que 9 (hiponatremia, infecção, retinopatia, hipoalbuminemia, persistência do canal arterial, desconforb respiratório precoce, baixo peso, prematuridade e peso por categorias) se mantiveram estatisticamente diferentes $(p<0,01)$ após análise por regressão logística. Quando analisadas por categoria de peso de nascimento, nenhuma variável demonstrou significância estatística. $O$ estudo sugere que o peso de nascimento é o maior fator - provavelmente o único - associado com gravidade da LSB cerebral neonatal.

PALAVRAS-CHAVE: recém nascido, lesão da substância branca cerebral, leucomalácia periventricular, infarto da substância branca periventricular, peso de nascimento.

\section{Birth weight as predictor for the severity of neonatal brain white matter lesion}

\begin{abstract}
To analyze the association of natal factors with the severity of neonatal brain white matter lesion (WML) by controlling the birth weight, we identified newborns with WML who were divided into: those with WML evolution towards resolution of the ultrasound image (less severe), and those who evolved with cist formation and/or ventriculomegalia and/or hemorrhage (greater severity). There were differences among the twelve variables (hyponatremia, anemia, infection, retinopathy, broncopulmonary dysplasia, hypoalbuminemia, persistence of the arterial canal, altered audiometry, early respiratory distress, birth weigh below $2,500 \mathrm{~g}$, weight per category, and prematurity) between the two groups $(p<0.05)$, being that nine variables (hyponatremia, infection, retinopathy, hypoalbuminemia, persistence of the arterial canal, early respiratory distress, low weight, prematurity, and weight per category) remained statistically different $(p<0.01)$ after the logistic re g ression analysis. When the variables were analyzed by birth weight category none of them presented statistical significance. This study suggests that birth weight is the major factor likely the only one - associated to the severity of neonatal brain white matter lesion.
\end{abstract}

KEY WORDS: newborn, brain white matter lesion, periventricular leukomalacia, periventricular white matter infarction, birth weight.

A lesão da substância branca (LSB) é a lesão cerebral mais freqüente no neonato pré-termo e a ter$\mathrm{mo}^{1}$, representadas pela leucomalácia (LPV) e pelo infartovenoso hemorrágico periventriculares (IVHP). Ambos são diagnósticos neuropatológicos.

Tanto a LPV, quanto o IVHP correlacionam-se com ecodensidade aumentada e/ou ecolucência periven- tricular na ultra-sonografia de crânio no período neonatal ${ }^{2,3}$. Contudo, a distinção ultra-sonográfica entre as duas lesões é difícil, pois ambas se manifestam por ecodensidade aumentada periventricular e evolução para ecolucência ${ }^{4}$, além de estarem freqüentemente associadas em um mesmo recém nascido ${ }^{2,5,6}$. Ecodensidade aumentada e/ou ecolucência têm sido relacio-

\footnotetext{
${ }^{1}$ Neu ropediatra, Mestre em Assistência Materno-Infantil, Professor de Neurociências da Faculdade Ruy Barbosa e Doutorando do Curso de Pós-graduação em Medicina e Saúde da Universidade Federal da Bahia, Salvador, Brasil (UFBA); ${ }^{2}$ Mestre em Saúde Comunitária, Doutor em Medicina, Pesquisador IA do CNPq; ${ }^{3}$ Neonatologista Intensivista; ${ }^{4}$ Psicólogo.
}

Recebido 30 Setembro 2005, recebido na forma final 23 Novembro 2005. Aceito 2 Dezembro 2005.

Dra. Nayara Argollo - Laboratório de Neurociências, Curso de Psicologia da Faculdade Ruy Barbosa - Rua Theodomiro Batista 42241940-320 Salvador BA - Brasil. E-mail: nayaraargollo@uol.com.br 
nadas com subseqüentes seqüelas neurológicas por vários autore $\mathrm{s}^{5,7-15}$, mas, é baixa a sensibilidade (22\%) do ultra-som de crânio para detectar as manifestações menores, somente diagnosticadas microscopicamente. Por outro lado, a alta especificidade, $92 \%{ }^{16}$, está arrolada à perda de parênquima cerebral visualizada ao ultra-som pela presença da ecolucência e ventriculomegalia (atrofia da substância branca). Desta forma, sinais de perda - ecolucência, ventriculomegalia - ou de compressão do parênquima cerebral - hidrocefalia, he mo rragias intraventriculares - ao ultra-som de crânio neonatal, prognosticam pobre evolução neurológica ${ }^{13}$.

Neste sentido, em várias investigações, fatores pré-natal, perinatal e pós-natal associam-se à gravidade da LSB. No entanto, todos os fatores referidos pelos respectivos investigadores também relacionamse ao baixo peso de nascimento ( $<2500$ gramas) e à sua principal causa, a prematuridade.

Partindo da hipótese de que todos os fatores associados com maior gravidade da LSB diagnosticada pela ultra-sonografia de crânio são epifenômenos do peso de nascimento, este estudo tem como objetivo analisar a associação entre fatores pré-natais, perinatais e pós-natais com a evolução desfavorável da LSB cerebral neonatal, controlando o peso de nascimento.

\section{MÉTODO}

Trata-se de estudo clínico-epidemiológico com base em dados secundários, realizado no período de janeiro de 1994 a dezembro de 2001, em uma maternidade da cidade de Salvador-Bahia. O hospital terciário selecionado, referência para gestações de alto risco em pacientes conveniadas e particulare, tem elevado número de partos por ano (2900 partos/ano) e o maior número de leitos em unidade de tratamento intensivo neonatal (UTIN) no Estado da Bahia (média de 340 internações /ano). O ultra-som craniano é realizado, sistematicamente, em todos os neonatos i n t e rnados na UTIN nos pri meiros 3 dias de vida, e no $7^{\circ}$, $14^{\circ}$ e $21^{\circ}$ dias de vida ou após identificação de intercorrências clínicas.

Através do laudo de ultra-som de crânio foram identificados todos os neonatos com LSB e seus prontuários foram revisados e anotados as intercorrências, diagnósticos e resultados dos exames complementares do período pré-natal, perinatal e pós-natal através de roteiro padronizado. Foram excluídos aqueles sem peso de nascimento. Foram anotados os escores do Apgar para o $1^{\circ}$ e o $5^{\circ}$ minutos, os resultados dos exames complementares analisados com base na variação do "normal" para essa faixa etária, coletada a idade gestacional baseada na data da última menstruação e, na ausência desta, pelo escore do Capurro. O diagnóstico de sofrimento fetal foi baseado na informação dada pelo obstetra na sala de parto, em resultados do Doppler ou ultrasom gestacionais, realizados quando havia suspeita clínica de sofrimento fetal (alteração da freqüência cardíaca fetal, desproporção de peso em gêmeos, líquido amniótico meconial, diminuição da movimentação fetal, parada de ganho de peso). No total, 106 variáveis foram identificadas para exploração de preditores e as quantitativas foram transformadas em dicotômicas (sim/não, ausente/presente), exceto peso de nascimento, idade materna, tempo de internação e idade gestacional, que foram re-categorizadas por faixas. A variável 'asfixia' correspondeu à presença deste diagnóstico na folha de problemas, ou entre os diagnósticos do neonato. O tempo de internação foi classificado a cada mês de internamento até 6 meses. O peso de nascimento foi categorizado em: 1) <999g; 2) 1000g-1499g; 3) 1500g2000 ; 4) 2001-2499g; e 5) $\geq 2500$; e o grau de prematuri dade em: 1) <32 semanas; 2) 32,0 a 33,6 semanas; 3) 34,0 a 36,6 semanas. O neonato com idade gestacional entre 37 semanas a 41 semanas e 6 dias foi considerado a termo.

As imagens das ultra-sonografias foram revisadas e classificadas pelas autoras (Argollo $\mathrm{N}$ e Ribeiro S). O critério de classificação para a LSB foi: 1) ecodensidade aumentada na região periventricular; 2) ecolucência isolada ou associada com ecodensidade e; 3) ecodensidade e/ou ecolucência complicada com ventriculomegalia (e/ou colpocefalia) e/ou hidrocefalia (com ou sem hemorragia intraventricular). Com esta classificação evita-se os termos anátomo-patológicos de leucomalácia periventricular e infarto hemorrágico periventricular, patologias de difícil diferenciação ao USC ${ }^{6}$, ao mesmo tempo que gradua-se a gravidade da perda de tecido cerebral, da ecodensidade aumentada (edema sem perda ce rebral), a ecolucência (necrose focal secundária a leucomalácia ou infarto hemorrágico periventricular), ambas podendo estar complicadas com atrofia (ventriculomegalia) ou compressão da substância branca (hidrocefalia, hemorragia). Para análise da gravidade foram constinuidos dois grupos: 1) de menor gravidade: ecodensidade periventricular isolada; 2) de maior gravidade: ecolucência e complicada. Para alocação nos grupos, foi escolhido o pior resultado de uma série de ultra-sons realizados no neonato. A época da lesão foi caracterizada em: 1) antenatal, se as ecolucências periventriculares tivessem sido detectados imediatamente após o nascimento; 2 ) perinatal, se a ecodensidade aumentada periventricular estavesse ausente logo após o nascimento, mas presente algumas horas após o nascimento e ecolucência identificada em torno de duas semanas do nascimento e 3) pós-natal, se as alterações aparecessem após evento clínico conhecido ${ }^{14}$. Como a região periventricular é normalmente hiperecogênica, só foi considerado patológico o aumento da ecodensidade periventricular de natureza globular (fla reperiventricular) cujo sinal fosse igual ou maior que o do plexo coróide ${ }^{15}$.

A análise dos dados foi realizada em duas fases. Na primeira fase, os dois grupos foram comparados entre si e identificadas as variáveis que estavam associadas a risco aumentado de maior gravidade da LSB. Na segunda, os pacientes foram estratificados por categoria de peso de nascimento, e todas as variáveis foram novamente comparadas. Para comparação dos grupos nas duas fases, foram utilizados testes estatísticos não-paramétricos: Mann-Whitney, para as variáveis quantitativas e qui-quadrado (Fisher, quando indicado) para as qualitativas. Os grupos cujas variáveis 


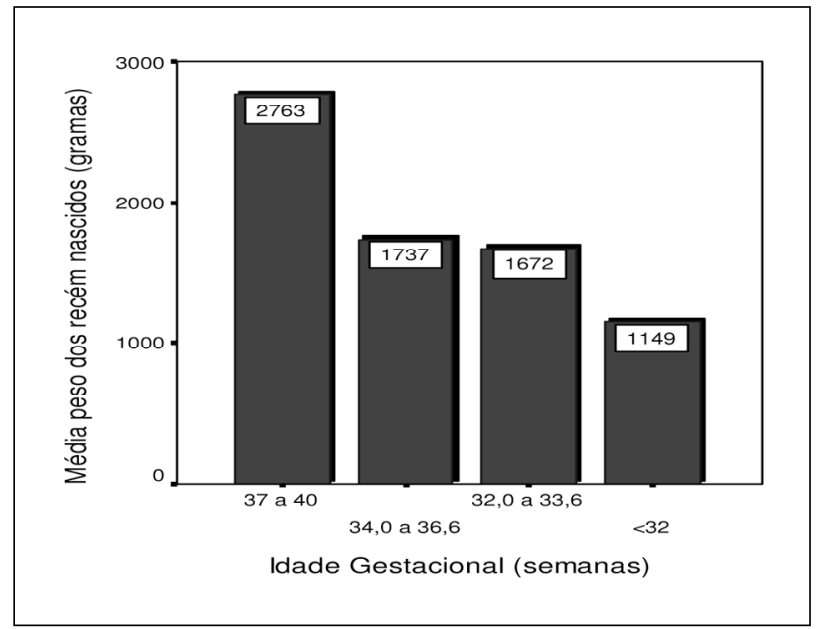

Gráfico 1. Distribuição das médias dos pesos por idade gestacio nal nos neonatos.

tinham mais de duas categorias foram comparados pelo Teste de Kruskal Wallis, e pós-teste Dunnett's T3. Para as variáveis quantitativas, foram estimadas a média com desvio-padrão, e a mediana com variação foi apresentada. Todas as variáveis com valor de $p<0,05$ da análise bivariada foram ponderadas por re gressão logística binária (fonvard stepwise) na segunda fase, para determinação de preditores independentes. Como múltiplas comparações estavam sendo realizadas, a significância estatística foi considerada para o valor de $p<0,01$, bicaudal, para diminuir o erro tipo 1 .

O projeto foi aprovado pelo Comitê de Ética em Pesquisa da Maternidade Climério de Oliveira/Curso de Pósgraduação em Medicina e Saúde da Universidade Federal da Bahia.

\section{RESULTADOS}

No período estudado, houve 2688 admissões na
UTIN e 93 (3,46\%) neonatos apresentaram sinais de LSB ao ultra-som de crânio, complicadas ou não com hemorragia intraventricular, hidrocefalia ou ventriculomegalia; 4 casos foram excluídos porque não havia registro do peso de nascimento.Foram realizadas, em média, 4,82 $\pm 3,45$ ultra-sons (mediana $=4,00$, variação: 1 a 22) em cada recém nascido.

Entre os 89 neonatos, 12 (13,5\%) foram a termo e, destes, 8 tiveram peso de nascimento superior a 2500g; entre os pre te rmos, 11 (12,4\%) tiveram peso nesta faixa. A prematuridade foi a principal causa do baixo peso de nascimento em 77 (86,52\%); 3 pretermos tiveram peso de nascimento superior a $2500 \mathrm{~g}$ (2500g, 2765g, 2860g), 2 entre 2000-2499g (2050g, $2190 \mathrm{~g})$ e, 4 a termo, com peso inferior a $2500 \mathrm{~g}$ (1870, 2024, 2025, e 2090 gramas, respectivamente) (Gráfico 1). A média do peso nascimento da amostra foi de 1502,52 $\pm 686,90$ (mediana: 1320 gramas, variação: 630-3495 gramas), sendo de 1228,76 \pm 775 , 98 (mediana: 1570 gramas, variação $750-3495$ gramas) no grupo de menor gravidade e, 1164,13 $\pm 331,99$ (mediana: 1197,50 gramas, variação: $630-1.800$ gramas) no grupo de maior gravidade.

A Tabela 1 expõe as características clínicas e demográficas da amostra e pode-se observar que as dife renças das médias entre as idades gestacionais, peso de nascimento e duração da internação foram significativamente diferentes entre os grupos de menor (ecodensidade aumentada) e maior (ecolucência e complicada) gravidade, e essa diferença se manteve significante após a regressão logística.

Sete $(6,6 \%)$ dentre as 106 variáveis relacionadas à história gestacional, parto, período perinatal e pós-

Tabela 1. Características dos grupos de gravidade pela alteração ultra-sonográfica.

\begin{tabular}{|c|c|c|c|c|}
\hline \multirow[b]{2}{*}{ Características } & \multicolumn{4}{|c|}{ Alteração ultra-sonográfica } \\
\hline & $\begin{array}{l}\text { Ecodensidade } \\
\text { aumentada } \\
\quad(n=49)\end{array}$ & $\begin{array}{l}\text { Ecolucência/ } \\
\text { complicado } \\
\quad(n=40)\end{array}$ & $\begin{array}{l}\text { Análise } \\
\text { univariada } \\
\text { Valor } p\end{array}$ & $\begin{array}{l}\text { Regressão } \\
\text { Logística Binária } \\
\text { Valor } p\end{array}$ \\
\hline $\begin{array}{l}\text { Idade materna (anos), média } \pm \text { DP } \\
\text { mediana (variação) }\end{array}$ & $\begin{array}{c}27,43 \pm 6,17 \\
29(14 \text { a } 38)^{1}\end{array}$ & $\begin{array}{c}28,62 \pm 6,11 \\
28,5(15-41)^{2}\end{array}$ & $0,467^{3}$ & \\
\hline $\begin{array}{l}\text { Idade gestacional (sem), média } \pm \text { DP } \\
\text { mediana (variação) }\end{array}$ & $\begin{array}{c}32,87 \pm 4,08 \\
33,00(25-40)\end{array}$ & $\begin{array}{c}28,96 \pm 2,66 \\
29,50(24-34)\end{array}$ & $0,00001^{3}$ & 0,00001 \\
\hline Sexo feminino (\%) & $\begin{array}{c}26 \\
(53,1)\end{array}$ & $\begin{array}{c}21 \\
(52,5)\end{array}$ & $0,958^{4}$ & \\
\hline $\begin{array}{l}\text { Peso de nascimento (gramas), média } \pm \text { DP } \\
\text { mediana (variação) }\end{array}$ & $\begin{array}{c}1.228,76 \pm 775,98 \\
1.570,00(750-3495)\end{array}$ & $\begin{array}{c}1.164,13 \pm 331,99 \\
1.197,50(630-1.800)\end{array}$ & $0,0005^{3}$ & 0,00001 \\
\hline $\begin{array}{l}\text { Internação (dias), média } \pm \mathrm{DP} \\
\text { mediana (variação) }\end{array}$ & $\begin{array}{c}32,8 \pm 26,37 \\
29,00(4 \text { a } 124)\end{array}$ & $\begin{array}{l}66,45 \pm 39,21 \\
62,00(6-225)\end{array}$ & $0,00028^{5}$ & 0,00001 \\
\hline
\end{tabular}

${ }^{1} n=42 ;{ }^{2} n=34 ;{ }^{3}$ teste de Mann Whitney; ${ }^{4}$ teste do qui-quadrado (Fisher); ${ }^{5}$ teste Kruskal Wallis. 
Tabela 2. Patologias pré-natal, perinatal e pós-natal entre neonatos com LSB.

\begin{tabular}{|c|c|c|c|c|}
\hline \multirow[b]{2}{*}{ Patologias } & \multicolumn{4}{|c|}{ Achado ao ultra-som } \\
\hline & $\begin{array}{c}\text { Grupo } \\
\text { ecodensidade } \\
\text { aumentada }(\%)\end{array}$ & $\begin{array}{c}\text { Grupo } \\
\text { ecolucência/ } \\
\text { complicada (\%) }\end{array}$ & $\begin{array}{c}\text { Análise } \\
\text { bivariada } \\
\text { Valor } \mathrm{p}^{1}\end{array}$ & $\begin{array}{c}\text { Regressão } \\
\text { logística binária } \\
\text { Valor } p\end{array}$ \\
\hline Hiponatremia & $4(8,2)$ & $12(30)$ & 0,01 & 0,0076 \\
\hline Anemia & $31(63,3)$ & $33(82,5)$ & 0,04 & 0,0446 \\
\hline Infecção & $28(57,1)$ & $35(87,5)$ & 0,002 & 0,0017 \\
\hline Retinopatia & $6(12,2)$ & $22(55)$ & 0,00001 & 0,00001 \\
\hline Displasia broncopulmonar & $5(10,2)$ & $12(30)$ & 0,018 & 0,0181 \\
\hline Hipoalbuminemia & $4(8,2)$ & $12(30)$ & 0,01 & 0,0076 \\
\hline Persistência do canal arterial & $1(2)$ & $10(25)$ & 0,002 & 0,0011 \\
\hline Audiometria alterada & $3(6,1)$ & $9(22,5)$ & 0,031 & 0,0244 \\
\hline Desconforto respiratório precoce & $35(71,4)$ & $40(100)$ & 0,00016 & 0,0002 \\
\hline Peso de nascimento $<2500$ gramas & $38(77,6)$ & $40(100)$ & 0,001 & 0,0014 \\
\hline Peso categorizado & $17(34,6)$ & $0(0)$ & $0,0003^{2}$ & 0,00001 \\
\hline Prematuro & $29(59,1)$ & $39(97,5)$ & 0,0004 & 0,0008 \\
\hline
\end{tabular}

${ }^{1}$ teste qui-quadrado (Fisher, quando indicado); ${ }^{2}$ teste Kruskal Wallis.

Tabela 3. Peso de nascimento (gramas) nos grupos de gravidade.

\begin{tabular}{lcccccc}
\hline & \multicolumn{5}{c}{ Peso (\%) } \\
\cline { 2 - 6 } Alteração ultra-sonográfica & $<999$ & $1.000-1.499$ & $1.500-1.999$ & $2.000-2.499$ & $>2.500$ & Total \\
\hline Ecodensidade & 6 & 16 & 10 & 6 & 11 & $(100)$ \\
aumentada & $(27,3)$ & $(50,0)$ & $(55,6)$ & $(100)$ & $(54,8)$ & 49 \\
Ecolucência/ & 16 & 16 & 8 & 0 & $(0,0)$ & $(45,2)$ \\
Complicada & $(72,7)$ & $(50,0)$ & $(44,4)$ & $10,0)$ & 11 & 89 \\
Total & 22 & 32 & $(10)$ & $(100)$ & $(100)$ & $(100)$ \\
\hline
\end{tabular}

VValor do $p$ pelo teste Kruskal Wallis entre os grupos: 0,00034; ${ }^{2}$ Valor do $p$ pelo teste Mann Whitney: grupo 2.000-2.499 X: <999g: 0,002; 1000-1499g: 0,025; 1500-1999: 0,049. grupo >2.500g X: <999g: 0,0001; 1000-1499g: 0,0203; 1500-1999: 0,011.

natal, tiveram diferenças estatisticamente significantes $(p<0,01)$. Doze delas foram analisadas pela regressão logística, porque tiveram valor do $p<0,05$, sendo que 9 se mantiveram significantes após a regressão: p resença de hiponatremia, infecção, retinopatia, hipoalbuminemia, persistência do canal arterial, desconforto respiratório precoce, baixo peso, prematuridade e peso por categorias (Tabela 2).

Quando a gravidade foi analisada por peso de nascimento, só houve diferença estatisticamente significante entre as categorias 2000-2499 gramas e >2500 gramas e as demais (Tabela 3). Os casos de maior gravidade diminuíram pro g ressivamente com o aumento do peso de nascimento e da idade gestacional. Não houve caso de maior gravidade entre os 17 neonatos com peso $\geq 2000$ gramas. Em relação à época da LSB, predominou o período perinatal, com 75 casos $(84,3 \%)$, sem diferenças significantes entre os grupos. Não houve neonatos no grupo de maior gravidade com peso $>2000 \mathrm{~g}$.

$\mathrm{Na}$ análise do grupo com peso de nascimento inferior a $\mathbf{2 0 0 0}$ gramas, nenhuma variável mostrou diferença estatisticamente significante entre os dois $g$ rupos de gravidade (Tabelas 4 e 5). O mesmo ocorreu na comparação dos grupos 2000-2499 gramas e $>2500$ gramas. 
Tabela 4. Características clínicas-demográficas por peso de nascimento e alteração ultra-sonográfica.

\begin{tabular}{|c|c|c|c|c|}
\hline Característica & $\begin{array}{c}\text { Peso } \\
\text { (gramas) }\end{array}$ & $\begin{array}{c}\text { Ecodensidade } \\
\text { aumentada }(n=49)\end{array}$ & $\begin{array}{c}\text { Ecolucência/ } \\
\text { Complicado }(n=40)\end{array}$ & Valor $p^{1}$ \\
\hline \multirow{7}{*}{$\begin{array}{l}\text { Idade gestacional, média } \pm \text { DP } \\
\text { (sem) } \\
\text { mediana (variação) } \\
\text { média } \pm \text { DP } \\
\text { mediana (variação) } \\
\text { média } \pm \text { DP } \\
\text { mediana (variação) } \\
\text { média } \pm \text { DP } \\
\text { mediana (variação) }\end{array}$} & $<999$ & $\begin{array}{c}27,83 \pm 1,94 \\
28,50(25-30)\end{array}$ & 26,95 & 0,294 \\
\hline & $1000-1499$ & $30,30 \pm 1,97$ & $29,63+1,86$ & 0,539 \\
\hline & & $29,65(28-34)$ & $30(26-32,4)$ & \\
\hline & 1500-1999 & $33,39 \pm 2,48$ & $31,65 \pm 1,40$ & 0,146 \\
\hline & & $33,5(30-37)$ & $31,6(30-34)$ & \\
\hline & $>2000$ & $36,76 \pm 3,05$ & a & \\
\hline & & $38(29-40)$ & & \\
\hline \multirow{7}{*}{$\begin{array}{l}\text { Peso de nascimento, média } \begin{array}{l}\text { (gramas) } \\
\text { mediana (variação) } \\
\\
\text { média } \pm \text { DP } \\
\text { mediana (variação) } \\
\text { média } \pm \text { DP } \\
\text { mediana (variação) } \\
\text { média } \pm \text { DP } \\
\text { mediana (variação) }\end{array}\end{array}$} & $<999$ & $\begin{array}{c}877,5 \pm 97,76 \\
867,5(750-990)\end{array}$ & $\begin{array}{c}821,56+105,16 \\
845,0(630-970)\end{array}$ & 0,329 \\
\hline & $1000-1499$ & $1219,38 \pm 131,34$ & $1272,50 \pm 130,59$ & 0,361 \\
\hline & & $1290(1005-1350)$ & $1290(1035-1490)$ & \\
\hline & 1500-1999 & $1653,5 \pm 107,03$ & $1632,5 \pm 89,72$ & 0,515 \\
\hline & & $1665(1500-1870)$ & $1630(1500-1800)$ & \\
\hline & $>2000$ & $2697,0 \pm 507,61$ & a & \\
\hline & & $2860(2024-3495)$ & & \\
\hline \multirow{8}{*}{$\begin{array}{l}\text { Internação (dias), média } \pm \mathrm{DP} \\
\text { mediana (variação) } \\
\text { média } \pm \mathrm{DP} \\
\text { mediana (variação) } \\
\text { média } \pm \mathrm{DP} \\
\text { mediana (variação) } \\
\text { média } \pm \mathrm{DP} \\
\text { mediana (variação) }\end{array}$} & $<999$ & $65 \pm 25,06$ & $90,63 \pm 50,43$ & $0,817^{3}$ \\
\hline & & $65(23-98)$ & $81(6-225)$ & \\
\hline & $1000-1499$ & $46,5 \pm 26,53$ & $54 \pm 16,46$ & $0,080^{3}$ \\
\hline & & $42(6-124)$ & $54(27-89)$ & \\
\hline & 1500-1999 & $27,20 \pm 8,75$ & $43 \pm 14,7$ & $1,00^{3}$ \\
\hline & & $30(15-42)$ & $46,5(24-61)$ & \\
\hline & $>2000$ & $11,82 \pm 11,12$ & a & \\
\hline & & $7(4-49)$ & & \\
\hline
\end{tabular}

'teste Mann Whitney; ${ }^{2}$ teste qui-quadrado; ${ }^{3}$ teste do Kruskal Wallis; aausência de caso, teste de significância não pôde ser realizado.

Tabela 5. Patologias do neonato por categoria de peso e alteração ultra-sonográfica.

\begin{tabular}{|c|c|c|c|c|}
\hline \multirow[b]{2}{*}{ Achados } & \multirow[b]{2}{*}{$\begin{array}{l}\text { Categoria do peso de } \\
\text { Nascimento (gramas) }\end{array}$} & \multicolumn{2}{|c|}{ Ultra-sonografia } & \multirow[b]{2}{*}{$\begin{array}{c}\text { Valor } \\
p^{1}\end{array}$} \\
\hline & & $\begin{array}{l}\text { Ecodensidade } \\
\text { aumentada (\%) }\end{array}$ & $\begin{array}{l}\text { Ecolucência/ } \\
\text { Complicada (\%) }\end{array}$ & \\
\hline Hiponatremia & $\begin{array}{c}<999 \\
1000 \text { a } 1499 \\
1500 \text { a } 1999 \\
>2000\end{array}$ & $\begin{array}{c}0(0) \\
2(12,5) \\
0(0) \\
2(11,8)\end{array}$ & $\begin{array}{c}6(37,5) \\
4(25) \\
2(25) \\
a\end{array}$ & $\begin{array}{l}0,133 \\
0,654 \\
0,183\end{array}$ \\
\hline Infecção & $\begin{array}{c}<999 \\
1000 \text { a } 1499 \\
1500 \text { a } 1999 \\
>2000\end{array}$ & $\begin{array}{c}4(66,7) \\
12(75) \\
7(70) \\
5(29,4)\end{array}$ & $\begin{array}{c}15(93,8) \\
13(81,3) \\
7(87,5) \\
a\end{array}$ & $\begin{array}{c}0,169 \\
1,00 \\
0,588\end{array}$ \\
\hline Retinopatia & $\begin{array}{c}<999 \\
1000 \text { a } 1499 \\
1500 \text { a } 1999 \\
>2000\end{array}$ & $\begin{array}{c}3(50) \\
2(12,5) \\
1(10) \\
0(0)\end{array}$ & $\begin{array}{l}12(75) \\
8(50) \\
2(25) \\
a\end{array}$ & $\begin{array}{l}0,334 \\
0,054 \\
0,559\end{array}$ \\
\hline Hipoalbuminemia & $\begin{array}{c}<999 \\
1000 \text { a } 1499 \\
1500 \text { a } 1999 \\
>2000\end{array}$ & $\begin{array}{c}1(16,7) \\
2(12,5) \\
0(0) \\
1(5,9)\end{array}$ & $\begin{array}{c}5(31,1) \\
4(25) \\
3(37,5) \\
a\end{array}$ & $\begin{array}{l}0,634 \\
0,654 \\
0,069\end{array}$ \\
\hline Persistência do canal arterial & $\begin{array}{c}<999 \\
1000 \text { a } 1499 \\
1500 \text { a } 1999 \\
>2000\end{array}$ & $\begin{array}{c}0(0) \\
1(6,3) \\
0(0) \\
0(0)\end{array}$ & $\begin{array}{c}4(25) \\
3(18,8) \\
3 \begin{array}{c}(37,5) \\
a\end{array}\end{array}$ & $\begin{array}{l}0,541 \\
0,600 \\
0,069\end{array}$ \\
\hline Desconforto respiratório precoce & $\begin{array}{c}<999 \\
1000 \text { a } 1499 \\
1500 \text { a } 1999 \\
>2000\end{array}$ & $\begin{array}{c}2(33,3) \\
13(81,3) \\
8(80) \\
1(5,9)\end{array}$ & $\begin{array}{c}3(18,8) \\
16(100) \\
8(100) \\
a\end{array}$ & $\begin{array}{l}0,585 \\
0,226 \\
0,477\end{array}$ \\
\hline Prematuro & $\begin{array}{c}<999 \\
1000 \text { a } 1499 \\
1500 \text { a } 1999 \\
>2000\end{array}$ & $\begin{array}{c}6(100) \\
16(100) \\
9(90) \\
6(35,3)\end{array}$ & $\begin{array}{c}16(100) \\
16(100) \\
8(100) \\
a\end{array}$ & $\begin{array}{c}\mathrm{b} \\
\mathrm{b} \\
1,00\end{array}$ \\
\hline
\end{tabular}

'teste qui-quadrado; aausência de caso, testes de significância não pode ser realizado; 'bprematuro é constante. 


\section{DISCUSSÃO}

$P$ rematuridade e peso de nascimento se relacionam indiretamente, e os neonatos mais prematuros são, também, os de menores pesos. Entre os recém natos da mesma idade gestacional, o risco de morbimortalidade perinatal é maior entre aqueles com menores pesos ${ }^{17,18}$. O peso de nascimento é, então, 0 maior preditor de morbidade e mortalidade no primeiroano de vida ${ }^{19}$, em particular no período neonatal. Por isso, e por ter maior fidedignidade de medida, neste trabalho, optou-se por comparar os grupos por peso de nascimento, e não por idade gestacional. Para avaliar a influência do peso de nascimento na gravidade da LSB, utilizou-se grupos compostos por neonatos pré-termo e a termo, onde a variável peso foi controlada na segunda fase da análise.

A freqüência da LSB diagnosticada pela ecodensidade e/ou ecolucência ao ultra-som de crânio varia de $8 \%$ a $89 \% 5,20$. Nos 89 neonatos avaliados com LSB, $45 \%$ tiveram sinais de perda e/ou compressão do parênquima cerebral, caracterizando condição desfavorável da doença. A ampla variação da freqüência detectada na literatura pode ser devida a diferenças metodológicas, critérios de classificação das lesões, ponto de corte do peso e instrumento utilizado para a neuroimagem (ultra-som, tomografiaouressonância).

No estudo de Larroque et al. ${ }^{20}$ ('EPIPAGE STUDY'), somente lesões císticas foram consideradas como de gravidade, sendo a ventriculomegalia, a hemorr a gia intraventricular e a hidrocefalia consideradas separadamente. Contudo, a associação com hemorragias intraventriculares, ventriculomegalia e hidrocefalia são fatores que agravam o prognóstico no neonato com LSB. Diferentemente do estudo EPIPAGE, que visava analisar as duas entidades separadamente (LSB e hemorragias intraventricular), Bass et al. ${ }^{5}$ e Kuban et al. ${ }^{6}$ encontraram freqüências mais altas, de $89 \%$ e $54,5 \%$, respectivamente, utilizando critérios diagnósticos diferentes para a alteração ultra-sonográfica. Todos esses estudos avaliaram, exclusivamente, neonatos na faixa de peso $<1500$ gramas ou de idade gestacional $<32$ semanas, faixas de maior risco de lesão grave. Em nosso estudo, a avaliação foi mais ampla, considerando previamente todas as faixas de peso e idade gestacional para detecção de fatores de risco para a gravidade da LSB. Pela mesma razão, os grupos de ecolucência e ecolucência complicadas foram analisados em conjunto.

Vários estudos apontam diferentes fatores perinatais associando-se com maior gravidade da LSB cerebral neonatal: displasiabroncopulmonar, transfusão de concentrado de hemácias ${ }^{21}$, infecção, hipotiroxinemia ${ }^{22}$, hipotensão $0^{23,24}$, baixo escore de Apgar no primeiro e quinto minutos, persistência do canal arterial, desconforto respiratório precoce, insuficiência respiratória ${ }^{25}$, pré-eclampsia ${ }^{26}$, hipocarbia ${ }^{27}$, hipematremia, cirurgias $^{28}$, enquanto anemia ${ }^{21}$, uso de corticóides pré-natal ${ }^{29}$ e pré-eclampsia ${ }^{22}$ seriam protetores. A maioria destes estudos, contudo, não controlou o peso de nascimento por faixas, ou, quando controlado, compararam somente o risco de desenvolver ecolucência (forma mais grave). No estudo de Dammann et al. ${ }^{30}$, dois grupos com e sem hipotensão arterial foram avaliados para a ocorrência de ecolucência, sem comparação com ecodensidade. Kubota et al. ${ }^{27}$ compararam recém nascidos com lesão cística cerebral (ecolucência ao ultra-som) nas diferentes faixas de peso em busca de fatores de risco. No presente estudo, entretanto, o grupo de menor gravidade (ecodensidade periventricular aumentada) foi comparado ao de maior gravidade (ecolucência e complicadas). A razão para esta distinção de grupos, é que, segundo a literatura, os sinais de perda de parênquima cerebral ao ultra-som (ecolucência, demonstrado por poros e atrofia da substância branca por ventriculomegalia) são mais graves, e assim, associando-se com pior prognóstico $5,7,9,10,16,25,31,32$. A hemorragia intraventricular, com ou sem hidrocefalia, dilata os ventrículos laterais, aumentando a compressão da substância branca periventricular, levando à isqüemia na área já em sofrimento hipóxico isquêmico ou agredida por citocinas inflamatórias. Neonatos com LSB têm 5 vezes mais chances de apresentar hemorragia intraventricular do que os neonatos sem $\mathrm{LSB}^{20}$, e as lesões mistas favorecem pobre prognóstico neurológico ${ }^{5}$.

O motivo de a maioria dos estudos avaliar somente grupos de neonatos com ecolucência deve-se à suposição que somente este achado ultra-sonográfico seria a expressão de LSB. Contudo, Bass et al. ${ }^{5}$ encontraram anormalidades neurodesenvolvimentais em crianças que tiveram ecodensidade persistente por duas ou mais semanas, enquanto Paneth et al..$^{33}$ supõem que deve haver significante LSB, mesmo quando não são observados cistos ao ultra-som.

No presente estudo, vários fatores estiveram significativamente associados $(p<0,01)$ ao grupo de maior gravidade: peso de nascimento, idade gestacional, hiponatremia, retinopatia, displasia broncopulmonar, hipoalbuminemia, persistência do canal arterial e desconforto respiratório precoce, após ponderação pela re g ressão logística (Tabelas 1 e 2). Contudo, nenhum, entre os 106 fatores analisados, demonstrou diferenças estatisticamente significantes entre os dois 
g rupos de gravidade quando foram re-comparados por faixa de peso (Tabelas 4 e 5), na segunda fase da análise. Este dado sugere que, muito provavelmente, somente o peso de nascimento é preditor da gravidade da LSB. Corroborando, nenhum caso de maior gravidade foi encontrado na faixa de peso $>2000$ gramas, e a percentagem de casos no grupo de maior gravidade foi aumentando progressivamente com a diminuição do peso. No estudo EPIPAGE, Larroque et al. ${ }^{20}$ obsenaram diminuição da incidência de ecolucência, de $10 \%$ para $2 \%$, da faixa de 27 semanas a 32 semanas de idade gestacional.

A duração da internação é considerada fator para ponderar a gravidade clínica dos recém nascidos. Neste estudo, a internação foi significantemente maior nos neonatos com maior gravidade da LSB (Tabela 1), mesmo quando categorizada a cada 30 dias, para evitar-se o efeito dos valores extremos. No entanto, quando comparada por faixa de peso, essa diferença desapareceu. Supõe-se que, entre neonatos com LSB, o peso é, também, o determinante do tempo de internação.

Este estudo apresenta várias características importantes: primeiro, avaliou grande número de fatores (106 variáveis) e controlou o peso de nascimento. Segundo, todos os 111 fatores foram novamente analisados quando o peso foi controlado. Apesar disso, algumas considerações devem ser feitas: 1) em estudos clínicos-epidemiológicos, variáveis confundidoras (outras que não o peso) podem estar distribuídas de fo rma desigual entre os diferentes grupos, e podem não ter sido identificadas neste estudo; 2 ) estudos descritivos estabelecem associações e não causalidade; e, 3) abrangeu grande período (8 anos), em área da medicina que está em constantes mudanças médicas e tecnológicas. Desta forma, fatores importantes como uso de corticóides na gestante para favorecer o amadurecimento pulmonar do prematuro e o uso de su rfactante nos neonatos com doenças das membranas hialinas não puderam ser controlados, pois o período estudado - década de 90 - representou a transição para as novas condutas.

Em conclusão, o estudo sugere que o peso de nascimento é fator - provavelmente o único - associado com maior gravidade da LSB cerebral neonatal, contudo não se avaliou o fluxo sangüíneo cerebral, possivelmente preditor da gravidade da referida lesão. Estudos prospectivos controlando o peso de nascimento e avaliando o fluxo sangüíneo cerebral em grupos de diferentes gravidades se fazem necessários para confirmação.

\section{REFERÊNCIAS}

1. Rezaie P, Dean A. Periventricular leucomalacia, inflammation and white matter lesions within the developing nervous system. Neuropathology 2002; 22:106-132.

2. Trounce JQ, Fagan D, Levene MI. Intraventricular haemorrhage and periventricular leucomalácia: ultrasound and autopsy correlation. Arch Dis Child 1986; 61:1203-1207.

3. Stewart Al, Reynolds EOR, Hope PL, et al. Probability of neurodevelopmental disorders estimated from ultrasound appearance of brains of very preterm infants. Dev Med Child Neurol 1987;29:3-11.

4. Hesser U, Katz-Salamon M, Mortenson W, Flodmark O, Forsberg H. Diagnosis of intracranial lesions in very-low-birthweight infants by ultrasound: incidence and association with potential risk factors. Acta Paediatr 1997;419:16-26.

5. Bass WT, Jones MA, White LE, Montgomery TR, Aiello F 3rd, Karlowics MG. Ultrasonographic differential diagnosis and neurodevelopmental outcome of cerebral white matter lesions in premature infants. J Perinatol 1999;9:330-336.

6. Kuban KCK, All red EN, Dammann O, et al. Topography of cerebral white-matter disease of prematurity studied prospectively in 1607 verylow-birth-weight infants. J Child Neurol 2001;16:401-408.

7. Graziani LJ. Pasto MP, Stanley C, et al. Neonatal neurosonographic correlates of cerebral palsy in preterm infants. Pediatrics 1986;78: 88-95.

8. Costello AML, Hamilton PA, Baudin J, et al. Prediction of neurodevelopmental impairment at four years from brain ultrasound appearance of very low preterm infants. Dev Med Child Neurol 1988;30:711-722.

9. Bor M, Ouden L, Guit GL. Value of cranial ultrasound and magnetic resonance imaging in predicting neurodevelopmental outcome in preterm infants. Pediatrics 1992;90:196-199.

10. Pilu G, Gabrielli S, Perolo A, Sandri F, Bovicelli. The clinical significance of fetal isolated cerebral borderline ventriculomegaly: report of 31 cases and review of the literature. Ultrasound Obstet Gynecol 1999; 14: 320-326.

11. Pereira MR, Funayama CAR. Avaliação de alguns aspectos da aquisição e desenvolvimento da linguagem de crianças nascidas pré-termo. Arq Neuropsiquiatr 2004;62:641-648.

12. Oliveira LN, Lima MCMP, Gonçalves VMG. Acompanhamento de lactentes com baixo peso ao nascimento. A rq Neuropsiquiatr 2003;61: 802-807.

13. Hashimoto K, Hasegawa H, Kida Y, Takeuchi Y. Correlation between ne $\mathrm{u}$ roimaging and neurological outcome in periventricular leucomalacia: diagnostic criteria. Pediatr Int 2001;43:240-245.

14. Kumazaki K, Nakayama M, Sumida $Y$, et al. Placental features in preterm infants with periventricular leucomalacia. Pediatrics 2002;109: 650-655.

15. Barkovich AJ, Hallam D. Neuroimaging in perinatal hypoxic-isquemic injury. Ment Retard Dev Disabil Res Rev 1997;3:28-41.

16. Stewart AL, Rifkin L, Amess PN, et al. Brain structure and neurocog nitive and behavioural function in adolescents who were born very preterm. Lancet 1999; 353:1653-1657.

17. Zaw W, Gagnon R, Silva O. The risks of adverse neonatal outcome among preterm small for gestational age infants according to neonatal versus fetal growth standards. Pediatrics 2003;111:1273-1277.

18. Barros FC, Hurtly SRA, Victora CG, Kirkwood MA, Vaughan JP. Comparion of the causes and consequences of prematurity and intrauterine growth retardation: a longitudinal study in southern Brazil. Pediatrics 1992;90:238-244

19. Wise PH. The anatomy of a disparity in infant mortality. Annu Rev Public Health 2003;24:241-262.

20. Larroque B, Marret S, Ancel PY, et al. The EPIPAGE Study Group. White matter damage and intraventricular hemorrhage in very preterm infants: the EPIPAGE study. J Pediatr 2003;143:477-483.

21. Paul DA, Pearlman SA, Finkelstein MS, Stefano JL. Cranial sonography in very-low-birth-weight infants: do all infants need to be screened? Clin Pediatr 1999; 38:503-509.

22. Dammann O, Kuban KCK, Leviton A. Perinatal infection, fetal inflammatory response, white matter damage, and cognitive limitations in children born preterm. Ment Retard Devl Disabil Res Rev 2002;8: 46-50 
23. Pearlman JM. White-matter injury in the preterm infant: an important determination of abnormal neurodevelopment outcome. Early Hum Dev 1998; 53:99-120.

24. Cunningham S, Symon AG, Elton RA, Zhu C, McIntosh N. Intra-arterial blood pressure reference ranges, death and morbidity in very-lowbirth-weight infants during the first seven days of life. Early Hum Dev 1999; 56:151-165.

25. Whitaker AH, Feldman JF, Rossem RV, et al. Neonatal cranial ultrasound abnormalities in low birth weight infants: relation to cognitive outcome at six years of age. Pediatrics 1996;98:719-729.

26. Krägeloh-Mann I, Toft P, Lunding J, Andresen J, Pryds O, Lou HC. Brain lesions in preterm: origin, consequences and compensation. Acta Paediatr 1999;88:897-908.

27. Kubota H, Ohsone Y, Oka F, Sueyoshi T, Takanashi J, Kohno Y. Significance of clinical risk factors of cystic periventricular leucomalacia in infants with different birthweights. Acta Paediatr 2001;90:302-308.

28. Perrott S, Dodds L, Vincer M. A population-based study of prognostic factors related to major disability in very preterm survivors. J Perinatol 2003;23:111-116.

29. LeFlore JL, Salhab WA, Broyles S, Engle WD. Association of antenatal and postnatal dexamethasone exposure with outcomes in extremely low birth weight neonates. Pediatrics 2002;110:275-279.

30. Dammann O, All red EN, Kuban KCK, et al. Systemic hypotension and white-matter damage in preterm infants. Dev Med Child Neurol 2002; 44:82-90.

31. Aziz K, Vickar DB, Sauve RS, Etches PC, Pain KS, Robertson CMT. Province-based study of neurologic disability of children weighting 500 th rough 1249 grams at birth in relation to neonatal cerebral ultrasound findings. Pediatrics 1995;95:837-844.

32. Sullivan MC, McGrath MM. Perinatal morbidity, mild motor delay, and later school outcomes. Dev Med Child Neurol 2003;45:104-112.

33. Paneth N, Rudelli R, Monte W, Rodriguez E, Pinto J, Kairam R. White matter necrosis in very low birth weight infants: neuropathologic and ultrasonographic findings in infants surviving six days or longer. J Pediatr 1990;116:975-984. 